\title{
CS Research Square \\ Expression of heat shock protein D1 is up-regulated in canine mammary tumors
}

\section{Gurpreet Kaur}

Guru Angad Dev Veterinary and Animal Sciences University

BV Sunil Kumar ( $\nabla$ drbvskumar@gmail.com )

Guru Angad Dev Veterinary and Animal Sciences University https://orcid.org/0000-0003-2641-6859

Kuldip Gupta

Guru Angad Dev Veterinary and Animal Sciences University

\section{Research Article}

Keywords:

Posted Date: January 31st, 2022

DOI: https://doi.org/10.21203/rs.3.rs-1290594/v1

License: (1) This work is licensed under a Creative Commons Attribution 4.0 International License.

Read Full License 


\section{Abstract \\ Background-}

Mammary tumors are frequently seen in female dogs. Conventionally, these tumors are diagnosed by histopathology. Differentially expressed biomarkers could aid in non-invasive diagnosis of canine mammary tumors but their number is scanty. The present investigation focuses on assessing HSPD1 as a diagnostic biomarker of canine mammary tumors.

\section{Methods and results-}

Expression of HSPD1 transcript was analyzed by SYBR green based real time PCR (qRT-PCR). Gene encoding immuno-dominant region of HSPD1 was cloned using the expression vector PPROEX-HTa and expressed in a prokaryotic system. Upon purification by affinity chromatography, a 60KDa recombinant protein was obtained which was confirmed by Western blotting. Hyperimmune serum was raised against the rHSPD1 in mice and immunohistochemistry was standardized to assess expression of this protein in various histotypes of canine mammary tumors. Elevated level of HSPD1 mRNA expression (4.92 \pm 0.65 folds) was observed in canine mammary tumors as compared to the healthy mammary tissue. Further, HSPD1 protein expression in $80 \%$ of healthy tissues showed mild immunoreactivity whereas, moderate to high expression of HSPD1 was seen in $80 \%$ CMT tissue samples.

\section{Conclusions-}

The findings suggest that HSPD1 could act as molecular marker for the diagnosis of mammary tumors in canines and also to assess the response to these subjects to treatment.

\section{Introduction}

The ubiquity of mammary tumor is seen in humans, dogs and cats but is infrequent in other species [1]. Canine mammary tumors are the most common neoplasia that are prominently seen in unsprayed female dogs [2]. There are a plethora of factors that contribute to the threat of developing the mammary tumors which include breed, age, ovariectomy, exogenous hormonal exposure, diet and obesity [3]. Conventionally, CMT is diagnosed by histopathological examination of the stained tumor sections. However, differentially expressed biomarkers can also be used for more specific diagnosis. Hence, identification and characterization of CMT specific/associated biomarkers is greatly encouraged for specific diagnosis of this malady [4].

Heat shock proteins (HSP) are highly conserved proteins that serve as mediators of hyperthermia resistance $[5,6,7]$. These proteins act as molecular chaperones that help in correct folding of the nascent polypeptide chains, translocation of proteins across the membranes, and marking the denatured ones for 
degradation $[8,9]$. Apart from the canonical functions, HSP also play an important role in carcinogenesis, immune response and apoptosis $[6,10]$.

In animal cells, the 60KDa HSPD1, also known as HSP60, primarily resides in the mitochondria whereas in plants they are located in the chloroplast. However certain studies have elucidated that HSPD1 is also located in the cytosol, cell surface, extra cellular space and biological fluids [11, 12]. In human beings, the HSPD1gene is situated on chromosome 2q33.1 [13]. It is abundantly expressed in normal cells but its expression is further induced by stress factors $[14,15]$.

Higher HSPD1 mRNA and protein levels have been significantly associated with breast carcinogenesis in human beings and auto-antibodies against this chaperone have been found to be useful for the early diagnosis of breast cancer [16-19]. Elevated HSPD1 expression is also observed in astroglioma cells [20], colorectal cancer [21], cervical cancer [22], ovarian cancer [23] and prostate cancer [24]. Moreover, HSPD1 over-expression has also been reported in cellular infiltrate of canine mixed mammary carcinoma [25]. Scanty reports are available on the expression of HSPD1 in various other histotypes of CMT. Hence, we undertook this study to compare the HSPD1 mRNA and protein expression in different histotypes of CMT.

\section{Methods}

\section{Sample sources}

CMT samples $(n=20)$ were collected from 6-12 years old dogs with a median of 8.5 years, during surgeries executed in the Department of Veterinary Surgery and Radiology, GADVASU, Ludhiana, India. Healthy canine mammary tissues were also collected from age and breed matched dogs $(n=20)$. For the extraction of total RNA, tissue samples were collected in RNAlater ${ }^{\mathrm{TM}}$ and stored at $-20^{\circ} \mathrm{C}$. For histopathological and immunohistochemical analyses, tissue samples were collected in $10 \%$ Neutral Buffered formalin. The Institutional Animal Ethics Committee (IAEC), GADVASU approved the collection of tissue samples and experimentation on mice via memo no. GADVASU/2020/IAEC/53/14; dated 25/01/2020. Moreover, approval from the Institutional Biosafety Committee (IBSC) was also procured via memo no. IBSC/20/9 dated 14/01/2020 for recombinant DNA technology work.

\section{Histopathology}

Tissue samples collected in 10\%NBF were processed and stained with Hematoxylin and Eosin (H\&E). Classification and grading of the tumors were carried out according to WHO standards of classification and grading $[26,27]$.

\section{RNA isolation and cDNA synthesis}

Total RNA was extracted using TRIzol ${ }^{\mathrm{TM}}$ reagent (Life Technologies, USA) following the manufacturer's protocol. Genomic DNA contamination (if any) was removed using DNAse1. About $1 \mu \mathrm{g}$ of the isolated RNA was used for c-DNA synthesis using oligo dT primers and Bio-Rad iScript c-DNA synthesis kit (Bio- 
Rad Laboratories, USA). Further the synthesized c-DNA was confirmed using specific GAPDH primers (Figure not shown here).

\section{Real Time PCR (qRT-PCR)}

Relative expression of HSPD1 mRNA in tumorous and healthy canine mammary tissues was assessed by SYBR green based qRT-PCR. The specific primers targeting HSPD1 gene (CGGGAACTAGCCTAAGCC and GTTCTTCCCTTTGGCCCCAT), along with endogenous housekeeping control genes, RPS-19 (CCTTCCTCAAAAAGTCTGGG and GTTCTCATCGTAGGGAGCAAG) [28] and $\beta$-actin (CCGCGAGAAGATGACCCAGA and GTGAGGATCTTCATGAGGTAGTCGG) [29] and iTaq universal SYBR® green supermix (BioRad, USA) were used for the expression profiling. The validity of these housekeeping genes had been previously checked in cancer studies [6]. Primers of all the three genes were used at $0.25 \mu \mathrm{M}$ final concentration. Annealing and extension of all the three genes were carried out at $60^{\circ} \mathrm{C}$ and dissociation curves were generated between $65^{\circ} \mathrm{C}$ to $95^{\circ} \mathrm{C}$ to assess the specificity of the amplicons.

The percent efficiencies of the PCR amplification for each gene was calculated as, $E=\left(10^{\left.-1 / s^{-0 p e}-1\right)} \times 100\right.$, where slope was calculated for the semi-log regression curve plotted between log c-DNA (serially diluted cDNA samples) versus their threshold cycle (Ct) values [30]. For evaluating the fold change in HSPD1 mRNA expression between canine mammary tumor and healthy mammary tissues, the Ct values of HSPD1 gene and the geometric mean of the Ct values of RPS-19 and $\beta$ actin after 40 cycles of amplification were utilized [31]. The statistical analyses were made according to Livak and Schmittgen (2001) [32] using SAS version 9.3. Further, qPCR score $\left(2^{-\delta C t}\right)$ was also calculated for each sample to analyze the relationship between HSPD1 expression at mRNA and protein level.

\section{Cloning and sequencing of the canine HSPD1 gene}

Using the primer pick program of NCBI, primers AAC CAT GGA TAT GCT TCG ATT ACC CGC AGT (forward) AAC TCG AGA ACG TGG TTA ACA GAG AGG CCA (reverse) were designed to amplify the gene encoding HSPD1 (Spanning $1643 \mathrm{bp}$ ). Further, to proceed with directional cloning, the sites for $\mathrm{Ncol}$ and Xhol restriction enzymes were added at the 5 ' ends of both the primers. The primers were custom synthesized from Integrated DNA Technologies (IDT). Herculase II fusion DNA polymerase kit (Agilent, USA) was used to amplify the HSPD1 gene as per the manufacturer's instructions. $0.8 \mu \mathrm{M}$ of each primer was used in $25 \mu \mathrm{l}$ reaction, which annealed with the template at $56^{\circ} \mathrm{C}$. The product thus obtained was resolved on agarose gel (1.5\%) and was purified using Quick gel extraction and PCR purification kit (Thermo Fisher Scientific, USA) following user's guide.

Restriction enzyme double digestion (REDD) reaction was individually initiated for purified PCR product and circular pPROEX-HTa expression vector using Xhol and Ncol (New England Biolabs, UK) restriction enzymes. Both the digested vector and insert were ligated using T4 DNA ligase at $22^{\circ} \mathrm{C}$ and the ligated product was transformed into competent BL21DE3 cells and plated onto LB agar plates containing ampicillin $(100 \mu \mathrm{g} / \mathrm{ml})$. Following 16 hours incubation, off-white colonies containing the recombinant plasmids were collected and allowed to grow in LB broth supplemented with the ampicillin $(100 \mu \mathrm{g} / \mathrm{ml})$. 
Subsequently, plasmids were isolated by alkaline lysis method [33] and restriction double digestion was performed to confirm the accurate orientation of insert into the vector using $\mathrm{Ncol}$ and $\mathrm{Xhol}$ (New England Bio labs, UK) restriction enzymes. Stabs containing the positive clones were forwarded to University of Delhi, South Campus (UDSC), New Delhi for custom sequencing. The deduced sequence was then deposited to NCBI \& accession number was obtained.

\section{Heterologous expression of the recombinant HSPD1 (rHSPD1) and its purification}

A positive clone was cultured in bulk and subjected for IPTG induction ( $1 \mathrm{mM})$ and pelleted down after 6 hours of induction. Purification of recombinant protein was performed under denaturing conditions as per standardized lab protocol [6]. Further, purified HSPD1 recombinant protein was dialyzed in Snake Skin Dialysis tubing, 10K MWCO (Thermo-Scientific, USA) for the removal of any residual urea and other undesired contaminant proteins. Expression of rHSPD1 was assessed by SDS-PAGE [34] and to confirm the expressed recombinantHSPD1 protein, western blotting [35] was carried using commercially available anti-HSPD1 antibody.

\section{Raising hyperimmune sera against canine rHSPD1 in mice}

To raise hyperimmune sera against rHSPD1, 10 Swiss Albino mice (4 months old) were used. Firstly mice were acclimatized for 1 week and later used for immunization. Purified recombinant HSPD1 protein was mixed with equivalent amount of Freund's Complete Adjuvant (FCA) and was subcutaneously injected in

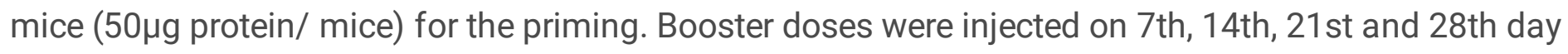
post priming with half the initial quantity of the protein emulsified in FIA. On 29th day, test bleeding was done from the tail veins of mice to examine the titer of antibodies [4]. The final bleeding was accomplished on 30th day by cardiac puncture and sera were collected. IgG purification kit (Thermo Scientific, USA) was used to purify immunoglobulin $\mathrm{G}$ from the harvested hyper immune sera. Western Blotting [39] was carried out using the purified IgG raised to detect HSPD1 in mammary tumor tissue homogenate.

\section{Immunohistochemistry (IHC) to analyze the tissue level expression of HSPD1}

$\mathrm{IHC}$ was carried out as per the protocol standardized in the lab [4]. Briefly, the tissue samples stored in $10 \%$ NBF were processed and paraffin wax blocks were prepared. Using rotary microtome $\otimes 5 \mu \mathrm{m}$ thick sections of tissues were cut and mounted on slides coated with poly-L lysine. Slides were firstly deparaffinized by 2 changes of xylene and then rehydrated by immersing them in graded alcohols. Further, heat induced retrieval of epitope was done by dipping the slides in citrate buffer and heating in microwave for varied time and watts ( 3 minutes at 850W, 7 minutes at 450W). Slides were cooled down to room temperature and washed with 3 changes of PBST. Subsequently endogenous blocking was carried out by mixing $35 \mu \mathrm{l}$ of $\mathrm{H}_{2} \mathrm{O}_{2}$ in $1000 \mathrm{ml}$ of methanol and incubated in humidify chamber for 30 minutes. In order to minimize non-specific binding 2.5\% Normal Horse Serum (Vector laboratories, USA) 
was added and incubated for 40 minutes. After the completion of incubation period, primary antibody (purified IgG from hyperimmune serum) was poured in 1:250 dilution, and in the negative control unimmunized mice serum was added and slides were incubated at $4^{\circ} \mathrm{C}$ for overnight.

On the subsequent day, secondary HRP conjugate antibody (ImmPRESS Universal polymer kit, peroxidase, Vector Laboratories, USA) was poured and the slides were incubated at room temperature for 30 minutes in humidify chamber. Impact DAB substrate kit (Vector Laboratories, USA) was used for color development. Then slides were counterstained with Gill's hematoxylin for 1 minute and were furthered dehydrated with graded alcohols and were finally mounted.

\section{Scoring of HSPD1 positive cells}

IHC analysis was carried out by applying semi-quantitative approach. In this method the intensity of developed brown color together with percentage of the cells showing positive staining were considered [4]. Scoring was carried out by a pathologist without considering the history of the patients [36]. To evaluate the $\mathrm{H}$ or SI score (staining index), the assigned score for the percentage of HSPD1 positive cells were multiplied with score provided for staining intensity. In order to detect the higher (SI>6) or lower $(S \mid<6)$ HSPD1 expression at tissue level, SI score was used. Percentage of the positive cells were calculated by randomly allotted scores as- 1 for $0-25 \%$ positive cells, 2 for $26-50 \%$ positive cells, 3 for 51 $75 \%$ positive cells and 4 for $76-100 \%$ positive cells. Staining intensity was scored as follows- no stating0 , mild staining- 1, moderate staining-2, intense staining- 3 respectively.

\section{Statistical Analysis}

Statistical analyses were performed with Statistical analysis software (SAS ver. 9.3). Pearson correlation coefficient ( $r$ ) was calculated between $\mathrm{H}$-score and $2^{-\delta C t}$ (qRT-PCR score) in order to determine any association between them. Spearman's rank correlation was also calculated between the scores. Statistical association of tumor histotypes with tumor grades, qRT-PCR score and $\mathrm{H}$ score, was assessed using the Kruscal Wallis test.

\section{Results}

\section{Histopathological analysis of CMT}

Of the 20 tumor samples, 11 were classified as complex carcinoma, three were anaplastic carcinoma, two each were papillary carcinoma and mixed tumor respectively, one each were hemangiosarcoma and mast cell tumor. About $55 \%(n=11)$ were designated as grade II (moderately differentiated), 30\% tumors $(n=6)$ were found to be of grade I (well differentiated) and only $15 \%$ tumors $(n=3)$ belonged to grade III (poorly differentiated).

Expression analysis of HSPD1 mRNA using Real Time PCR 
In our study, HSPD1, RPS19 and $\beta$-actin genes had amplification efficiencies of $86.78 \%, 95.63 \%$, and $96.44 \%$ respectively, suggesting that the exponential efficiencies of HSPD1 gene along with the internal controls were suitable for real time PCR. A significantly elevated expression of $4.92 \pm 0.65 f o l d s(p<0.05)$ for HSPD1 mRNA was observed in canine mammary tumors as compared with healthy tissue samples.

\section{Cloning, expression of HSPD1 gene and purification of recombinant HSPD1 protein}

A single specific band corresponding to $₫ 1643$ bp was resolved upon the PCR amplification using specific primers targeting the HSPD1 gene (Fig. 1). The isolated plasmids from the cultured positive clones, upon $\mathrm{Ncol}$ and $\mathrm{Xhol}$ restriction enzyme double digestion released a specified insert of 1643bp (Fig. 2), which confirmed proper cloning of the gene. The obtained positive clones were forwarded for custom sequencing and the deduced sequence was deposited to GenBank of National Center for Biotechnology information (NCBI) with an accession no. OK484370.

Upon induction with $1 \mathrm{mM}$ IPTG, the expression kinetics revealed that after 6 hours post IPTG induction, expression level of rHSPD1 was optimum. Upon Ni-NTA based affinity chromatography, under denaturing conditions, the purified recombinant protein was resolved as a $\triangle 60 \mathrm{kD}$ a protein on SDS-PAGE analysis (Fig. 3). The total yield of the rHSPD1 protein was $6.2 \mathrm{mg} / \mathrm{L}$ of the induced culture. A specified immunereactivity corresponding to $60 \mathrm{kDa}$ on the nitrocellulose membrane post Western blotting confirmed the recombinant protein (Fig. 4).

The IgG purified from the hyperimmune sera also distinctly reacted with native HSPD1 in the tumor tissue homogenate upon Western blotting (Fig. 5), which confirmed that the purified IgG specifically reacted with the native cellular HSPD1.

\section{Immunohistochemical detection of HSPD1 expression in canine mammary neoplasia}

The HSPD1immunopositive cells were stained brown, which was restricted to the cytoplasm of the cells alone. The negative controls, in which unimmunized mice serum was used, didn't show any immunostaining with the raised HSPD1 antibodies. Immunoreactivity against cellular HSPD1 was judged based on the staining index (SI) obtained by multiplying the number of immunopositive cells in random fields with the staining intensity score of the pathologist. Tissue expression of HSPD1was judged as strong when SI was greater than/equal to 6 and it was referred to as mild when SI was less than 6 [4]. Based on this convention, a mild expression of HSPD1 was detected in most $(80 \%)$ of the healthy mammary glands (Fig. 6), while a varied HSPD1 expression was evident in most of the CMT histotypes under study. The immunoreactivity for HSPD1 was found to be strong in $80 \%$ of the CMT tissues (Fig. 7). Further, a weak HSPD1 expression was indicated in all the specimens of hemangiosarcoma and papillary carcinoma, while, a strong HSPD1 expression was seen rest of the histotypes of CMT under study (mixed mammary tumors, anaplastic carcinomas complex carcinomas and mast cell tumors). When, HSPD1 expression was compared with different grades of CMT, a strong HSPD1 expression was discerned in $50 \%, 72.73 \%$ and $85.57 \%$ of grade- 1 , grade- 2 and grade- 3 CMT respectively. 


\section{Relationship between HSPD1 expression at mRNA and protein level in various types of CMT}

The statistical analysis between the IHC score and qRT-PCR score revealed that there was no significant correlation between HSPD1 transcript and protein expression. However, the Kruscal Wallis test revealed significant differences $(P<0.05)$ in the $\mathrm{H}$ scores alone with respect to different $\mathrm{CMT}$ histotypes. The Wilcoxon mean rank for $\mathrm{H}$ score was highest in mixed mammary tumor and least in papillary carcinoma indicating that HSPD1 expression was least in papillary carcinoma and was highest in mixed mammary tumors among the CMT histotypes under study. Moreover, one way ANOVA was also carried out to check for the significant differences of HSPD1 expression (if any) in various histotypes of CMT based on the $\mathrm{H}$ scores. It was found that, the expression of the protein (H-score) in complex carcinoma $(9.00 \pm 0.69)$, mixed mammary tumors $(10.00 \pm 0.91)$ and mast cell tumors $(8.00 \pm 1.29)$ were significantly higher $(P<0.05)$ than that in papillary carcinoma $(1.68 \pm 1.05)$.

\section{Discussion}

In this contemporary epoch, the incidence of canine mammary neoplasia, is raising day by day globally. But, with the advent of advanced technology, it is possible to diagnose a majority of these tumors at an early stage. For the diagnosis of CMT, the histopathological examination is valuable but involves an invasive tissue collection process and fine needle aspiration which is less invasive is less specific too. In this pursuit, the identification of certain biomarkers to diagnose this malady is absolutely crucial. The progressive research in the cancer biology has depicted the significance of HSP as potential biomarkers of carcinogenesis for certain human cancer types [6]. HSPD1 is a heat shock protein that also plays an indispensable role in immune responses has anti-apoptotic characteristics and promotes tumor growth, progression, invasion, and metastasis. It is also accountable for treatment resistance and worse survival rates. During carcinogenesis, HSPD1 is deposited on the outside of mitochondria, in the cytoplasm, plasma membrane, and secretory vesicles, protecting tumour cells from external environmental stress and promoting cell proliferation. HSPD1 also plays a role in the permeabilization of the mitochondrial membrane by interacting with cyclophilin $\mathrm{D}$, a protein that regulates the permeability transition pore in mitochondria. HSPD1 controls the action of p53 and causes a cytoprotective cascade by stabilising the quantum of survivin. The mitochondrial survivin stores are depleted as a result of acute HSPD1 extraction. Survivin is known for inhibiting apoptosis, as well as increasing p53 expression and triggering p53-dependent apoptosis in tumour cells. These cytoprotective characteristics of HSPD1 have been thoroughly investigated in vivo in malignancies, where HSPD1 is selectively upregulated in contrast to normal cells, while HSPD1 deficiency in normal cells is not connected to mitochondrial malfunction or cell death.

Moreover, HSPD1 not only stabilises the amount of survivin in the mitochondria, but it also provides cytoprotection through another method, which involves the formation of a complex by HSPD1's connection with p53, which inhibits the function of p53 tumor cells [37]. Several reports advocate that 
HSPD1 is up-regulated in human breast cancer. Provided that, CMT and human breast cancer share a close association, we chose to study the expression ofHSPD1 in canine mammary tumors.

In our study majority of the tumors were complex carcinomas. Many reports in the past also advocate that complex carcinomas, among all other canine mammary tumors are the most pervasive $[6,10,25,38$, $39,40,41]$. In our study, HSPD1 mRNA was $4.92 \pm 0.65$ folds overexpressed in CMT. In the past, Desmetz et al (2008) [16] reported an elevated expression of HSPD1 in early breast cancer. Apart from breast cancer, an increased HSPD1 mRNA expression has also been reported in ovarian [42] and colorectal cancer [43] in human beings. However, a down regulation of HSPD1 mRNA expression has also been reported in hepatocellular carcinoma [44].

In human breast pathology, IHC is routinely used in the diagnosis and prognosis of breast neoplasia. In the present study, we found a strong expression of HSPD1 in a majority of CMT tissues. In the past, a moderate to high cytoplasmic expression of HSPD1 has been reported in cellular infiltrate of mixed canine mammary carcinomas [25] and triple negative human breast cancer [45] which was associated with an advanced stage of tumor. It has also been reported that the elevated expression of HSPD1 in initial stages of breast carcinogenesis has a significant correlation with tumor growth and progression [16]. In or study, we found a strong HSPD1 expression in a majority of CMT histotypes. Hence, adding HSPD1 to the current panel of CMT biomarkers will certainly improve the sensitivity and specificity of CMT diagnosis.

\section{Conclusion}

We report an elevated level of HSPD1 expression in various histotypes of canine mammary tumors. HSPD1 protein expression was maximum in mixed mammary tumors whereas in the papillary carcinoma the expression was minimum. However, due to the smaller sample size, the data need to be confirmed in a study employing a large number and types of CMT in future.

\section{Declarations}

\section{Conflict of interest:}

None of the authors of this paper has a financial or personal relationship with other people or organizations that could inappropriately influence or bias the content of the paper.

\section{Ethics approval:}

This study was performed in line with the principles of the Institutional Animal Ethics Committee (IAEC). Approval was granted by the IAEC, GADVASU, India vide memo no. GADVASU/2020/IAEC/53/14; dated 25/01/2020.

\section{Consent to participate:}


This experiment was not conducted on human subjects. However, consent was obtained by the owners of the dogs to collect the samples for experimentation.

\section{Consent to publish:}

This experiment was not conducted on human subjects. However, consent has been obtained from the Director of Research of GADVASU, India for publishing this manuscript.

\section{Funding:}

The authors are very much thankful to the Department of Biotechnology, India, for rendering all the necessary funds (BT/ADV/Canine Health/GADVASU/2017-18 dated 27-03-2018) required for the accomplishment of the research work.

\section{References}

1. Goldschmidt MH, Peña L, Zappulli V (2016) Tumors of the mammary gland.Tumors Domest Anim723-765

2. Sleeckx N, De Rooster H, EJ VK, Van Ginneken C, Van Brantegem L (2011) Canine mammary tumours, an overview. Reprod Domest Anim 46(6):1112-1131

3. Schneider R, Dorn CR, Taylor DO (1969) Factors influencing canine mammary cancer development and postsurgical survival. J Natl Cancer Inst 43(6):1249-1261

4. Pandey M, Kumar BVS, Gupta K, Sethi RS, Kumar A, Verma R (2018) Over-expression of mammaglobin-B in canine mammary tumors. BMC Vet Res 14(1):1-8

5. Lindquist S (1986) The heat-shock response. Annu Rev Biochem 55(1):1151-1191

6. Kumar BVS, Bhardwaj R, Mahajan K, Kashyap N, Kumar A, Verma R (2018) The overexpression of Hsp90B1 is associated with tumorigenesis of canine mammary glands. Mol Cell Biochem 440(1):23-31

7. Calderwood SK (2010) Heat shock proteins in breast cancer progression-a suitable case for treatment? Int J Hyperthermia 26(7):681-685

8. Tutar L, Tutar Y (2010) Heat shock proteins; an overview. Curr Pharm Biotechnol 11(2):216-222

9. Weibezahn J, Schlieker C, Tessarz P, Mogk A, Bukau B (2005) Novel insights into the mechanism of chaperone-assisted protein disaggregate. J Biol Chem. https://doi.org/10.1515/BC.2005.086

10. Badowska-Kozakiewicz AM, Malicka E (2012) Immunohistochemical evaluation of expression of heat shock proteins HSP70 and HSP90 in mammary gland neoplasms in bitches. Pol J Vet Sci 15(2)

11. Jindal S, Dudani AK, Singh BH, Harley CB, Gupta RS (1989) Primary structure of a human mitochondrial protein homologous to the bacterial and plant chaperonins and to the 65-kilodalton mycobacterial antigen. Mol Cell Biol 9(5):2279-2283 
12. Cappello F, Conway de Macario E, Marasà L, Zummo G, Macario AJ (2008) HSP60 expression, new locations, functions, and perspectives for cancer diagnosis and therapy. Cancer Biol Ther 7(6):801809

13. Bukau B, Horwich AL (1998) The Hsp70 and HSP60 chaperone machines. Cell 92(3):351-366

14. Nakamura H, Minegishi H (2013) HSP60 as a drug target. Curr Pharm Des 19(3):441-451

15. Hansen JJ, Bross P, Westergaard M, Nielsen M, Eiberg H, Børglum AD, Mogensen J, Kristiansen K, Bolund L, Gregersen N (2003) Genomic structure of the human mitochondrial chaperonin genes: HSP60 and HSP10 are localised head to head on chromosome 2 separated by a bidirectional promoter. Hum Genet 112(1):71-77

16. Desmetz C, Bibeau F, Boissiere F, Bellet V, Rouanet P, Maudelonde T, Mangé A, Solassol J (2008) Proteomics-based identification of HSP60 as a tumor-associated antigen in early stage breast cancer and ductal carcinoma in situ. J Proteome Res 7(9):3830-3837

17. Seigneuric R, Mjahed H, Gobbo J, Joly AL, Berthenet K, Shirley S, Garrido C (2011) Heat shock proteins as danger signals for cancer detection. Front Oncol 1:37

18. Li DQ, Wang L, Fei F, Hou YF, Luo JM, Zeng R, Wu J, Lu JS, Di GH, Ou ZL, Xia QC (2006) Identification of breast cancer metastasis-associated proteins in an isogenic tumor metastasis model using twodimensional gel electrophoresis and liquid chromatography-ion trap-mass spectrometry. Proteomics 6(11):3352-3368

19. Bini L, Magi B, Marzocchi B, Arcuri F, Tripodi S, Cintorino M, Sanchez JC, Frutiger S, Hughes G, Pallini V, Hochstrasser DF (1997) Protein expression profiles in human breast ductal carcinoma and histologically normal tissue. Electrophoresis 18(15):2832-2841

20. Bajramović JJ, Geutskens SB, Bsibsi M, Boot M, Hassankhan R, Verhulst KC, van Noort JM (2000) The stress kit: a new method based on competitive reverse transcriptase-polymerase chain reaction to quantify the expression of human aB-crystallin, Hsp27, and HSP60. Cell Stress Chap 5(1):30

21. Mori D, Nakafusa Y, Miyazaki K, Tokunaga O (2005) Differential expression of Janus kinase 3 (JAK3), matrix metalloproteinase 13 (MMP13), heat shock protein 60 (HSP60), and mouse double minute 2 (MDM2) in human colorectal cancer progression using human cancer cDNA microarrays. Pathol Res Pract 201(12):777-789

22. Castle PE, Ashfaq R, Ansari F, Muller CY (2005) Immunohistochemical evaluation of heat shock proteins in normal and preinvasive lesions of the cervix. Cancer Lett 229(2):245-252

23. Schneider J, Jimenez E, Marenbach K, Romero H, Marx D, Meden H (1999) Immunohistochemical detection of HSP60-expression in human ovarian cancer. Correlation with survival in a series of 247 patients. Anticancer Res 19(3A):2141-2146

24. Johansson B, Pourian MR, Chuan YC, Byman I, Bergh A, Pang ST, Norstedt G, Bergman T, Pousette Ã (2006) Proteomic comparison of prostate cancer cell lines LNCaP-FGC and LNCaP-r reveals heatshock protein 60 as a marker for prostate malignancy. Prostate 66(12):1235-1244

25. Lopes-Neto BE, Souza SC, Bouty LM, Santos GJ, Oliveira ES, de Freitas JC, Nunes-Pinheiro DC (2017) CD4+, CD8+, FoxP3+ and HSP60+ expressions in cellular infiltrate of canine mammary carcinoma in 
mixed tumor. Acta Sci Vet 45:1-8

26. Goldschmidt M, Peña L, Rasotto R, Zappulli V (2011) Classification and grading of canine mammary tumors. Vet Pathol 48(1):117-113

27. Karayannopoulou M, Kaldrymidou E, Constantinidis TC, Dessiris A (2005) Histological grading and prognosis in dogs with mammary carcinomas: application of a human grading method. $\mathrm{J}$ Comp Pathol 133(4):246-252

28. Wang YS, Chi KH, Chu RM (2007) Cytokine profiles of canine monocyte-derived dendritic cells as a function of lipopolysaccharide-or tumor necrosis factor-alpha-induced maturation. Vet Immunol and Immunopathol 118(3-4):186-198

29. Timmermans-Sprang EP, Gracanin A, Mol JA (2015) High basal Wntsignaling is further induced by $\mathrm{PI} 3 \mathrm{~K} / \mathrm{mTor}$ inhibition but sensitive to cSRC inhibition in mammary carcinoma cell lines with HER2/3 overexpression. BMC Cancer 15(1):1-12

30. McNeill RE, Miller N, Kerin MJ (2007) Evaluation and validation of candidate endogenous control genes for real-time quantitative PCR studies of breast cancer. BMC Mol Biol 8(1):1-13

31. Abasht B, Kaiser MG, Van der Poel J, Lamont SJ (2009) Genetic lines differ in Toll-like receptor gene expression in spleens of chicks inoculated with Salmonella entericaserovarEnteritidis. Poult Sci 88(4):744-749

32. Livak KJ, Schmittgen TD (2001) Analysis of relative gene expression data using real-time quantitative PCR and the $2-\Delta \Delta C T$ method. Methods 25(4):402-408

33. Sambrook J, Russell DW (2001) Molecular cloning: a laboratory manual, vol 1. Cold Spring Harbor Laboratory, Cold Spring Harbor, NY, p 112

34. Laemmli UK (1970) Cleavage of structural proteins during the assembly of the head of bacteriophage T4. Nature 227(5259):680-685

35. Towbin H, Staehelin T, Gordon J (1979) Electrophoresis transfer of protein from polyacrylamide gels to nitrocellulose sheets. Procedure and applications. Proc Natl Acad Sci USA 76:4350-4354

36. Rizzardi AE, Johnson AT, Vogel RI, Pambuccian SE, Henriksen J, Skubitz AP, Metzger GJ, Schmechel SC (2012) Quantitative comparison of immunohistochemical staining measured by digital image analysis versus pathologist visual scoring. Diagn Pathol 7(1):1-10

37. Asea AA, Kaur P (eds) (2019) Heat Shock Protein 60 in Human Diseases and Disorders. Springer

38. Pandey M, Kumar BS, Verma R (2015) Mammaglobin as a diagnostic serum marker of complex canine mammary carcinomas. Res Vet Sci 103:187-192

39. Mulligan RM (1975) Mammary cancer in the dog: a study of 120 cases. Am J Vet Res 36(9):13911396

40. Mitchell L, De la Iglesia FA, Wenkoff MS, Van Dreumel AA, Lumb G (1974) Mammary tumors in dogs: survey of clinical and pathological characteristics. Can Vet J 15(5):131

41. Birdi R, Kumar BS, Gupta K, Kashyap N, Kumar A (2019) Circulating level of heat shock protein 27 is elevated in dogs with mammary tumors. 3 Biotech 9(6):1-9 
42. Abu-Hadid M, Wilkes JD, Elakawi Z, Pendyala L, Perez RP (1997) Relationship between heat shock protein 60 (HSP60) mRNA expression and resistance to platinum analogues in human ovarian and bladder carcinoma cell lines. Cancer Lett 119(1):63-70

43. Campanella C, Rappa F, Sciumè $C$, Marino Gammazza A, Barone R, Bucchieri F, David S, Curcurù G, Caruso Bavisotto C, Pitruzzella A, Geraci G (2005) Heat shock protein 60 levels in tissue and circulating exosomes in human large bowel cancer before and after ablative surgery. Cancer 121(18):3230-3239

44. Zhang J, Zhou X, Chang H, Huang X, Guo X, Du X, Tian S, Wang L, Lyv Y, Yuan P, Xing J (2016) HSP60 exerts a tumor suppressor function by inducing cell differentiation and inhibiting invasion in hepatocellular carcinoma. Oncotarget 7(42):68976

45. Bodoor K, Abu-Sheikha A, Matalka I, Alzou'bi H, Batiha O, Abu-Awad A, Jalboush SA, Fayyad LM, Qadiri E, Jarun Y, Albatayneh K (2018) Immunohistochemical analysis of heat shock proteins in triple negative breast cancer: HSP60 expression is a marker of poor prognosis. Eur $\mathrm{J}$ Gynaecol Oncol 39(6):926-934

\section{Figures}




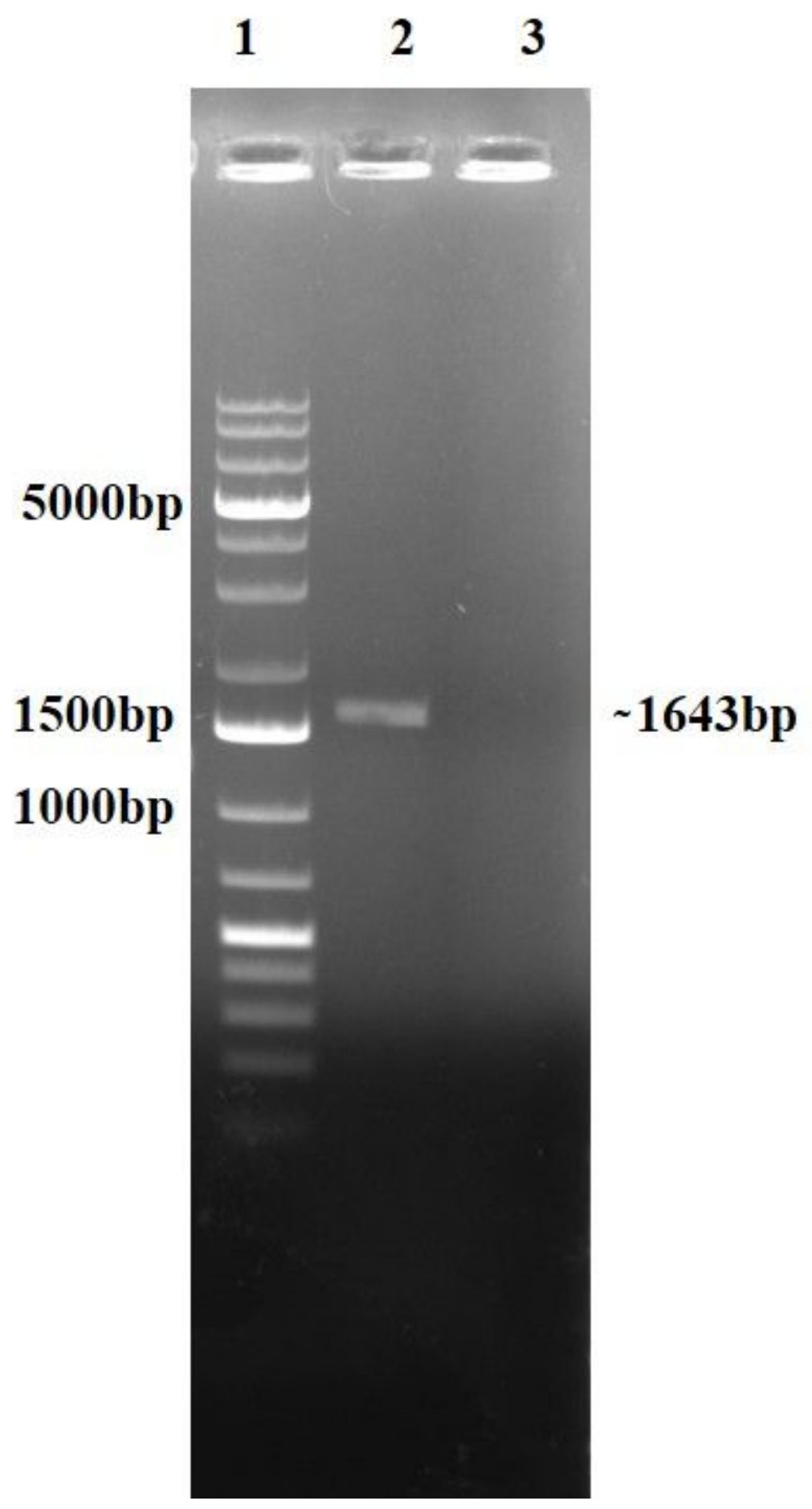

Figure 1

Amplicon of HSPD1 gene (Lane 1: 1kb plus DNA ladder; Lane 2: $\triangle 1643 \mathrm{bp}$ HSPD1amplicon; Lane 3: non template control (NTC) 


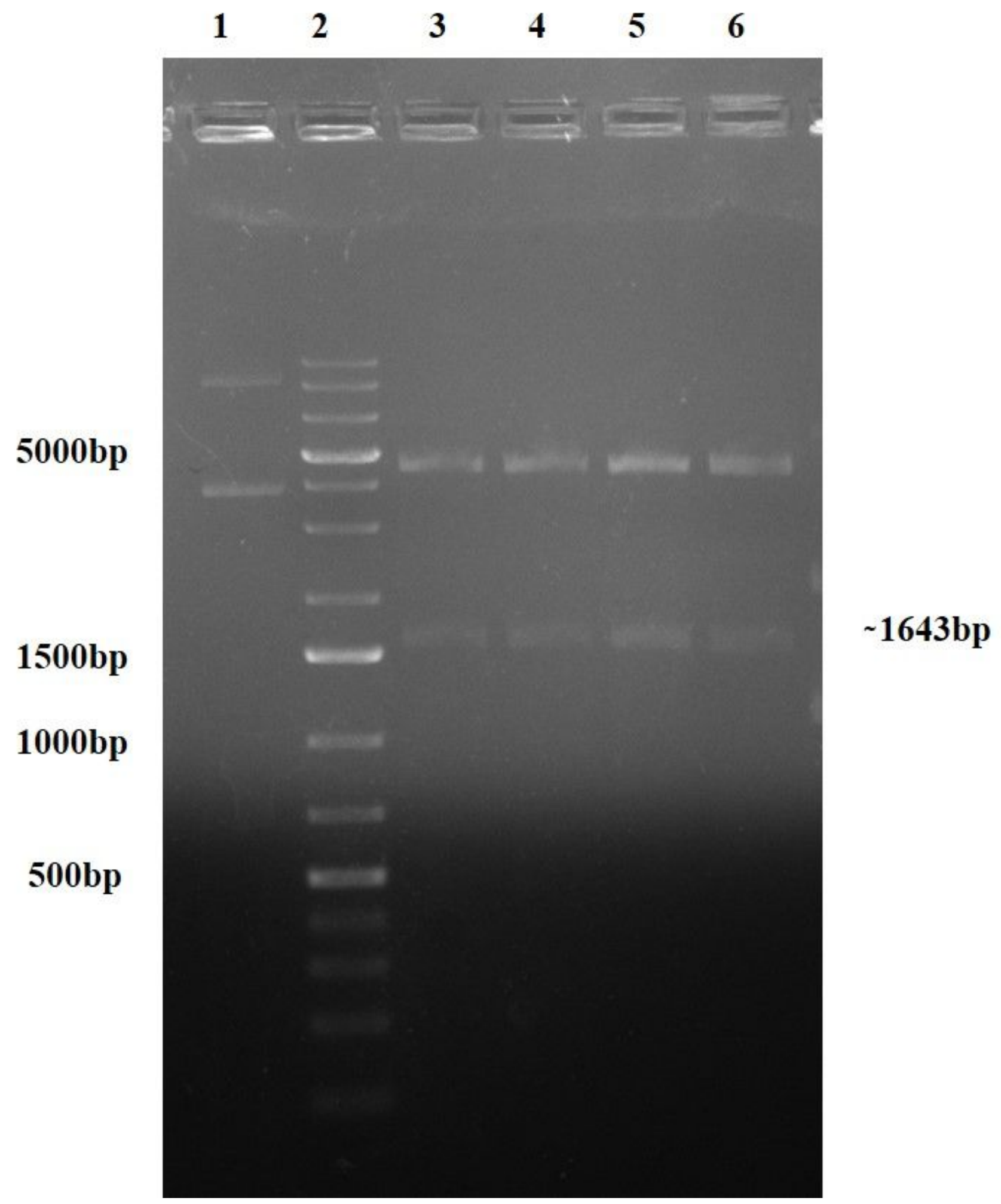

Figure 2

Restriction enzyme double digestion of pPROEXHTa- HSPD1plasmid (Lane 1: undigested plasmid;Lane 2: 1 kb plus DNA ladder;Lanes 3-6: Nco1 and Xho1 digested plasmid) 

M
1
2
3

$75 \mathrm{kDa}$

63kDa

$\sim 60 \mathrm{kDa}$

48kDa

35kDa

\section{Figure 3}

SDS PAGE depicting purified recombinant HSPD1 protein (Lane M: Pre- stained protein ladder;Lane 1-3: purified protein) 

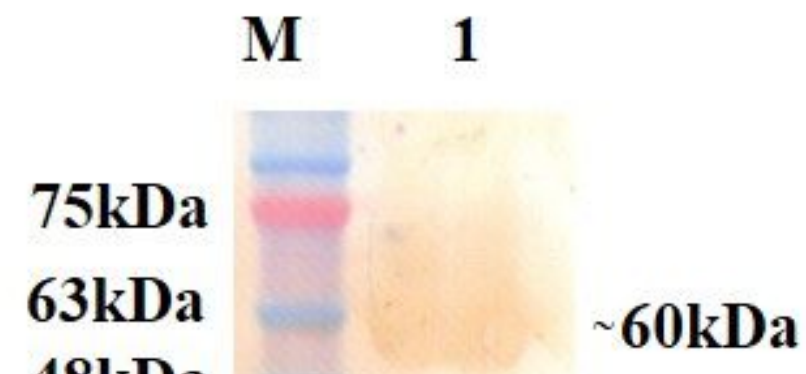
48kDa

35kDa

\section{Figure 4}

Western blotting analysis of recombinant HSPD1 protein (Lane M: Pre- stained protein ladder; Lane 1: Immuno reactive rHSPD1) 


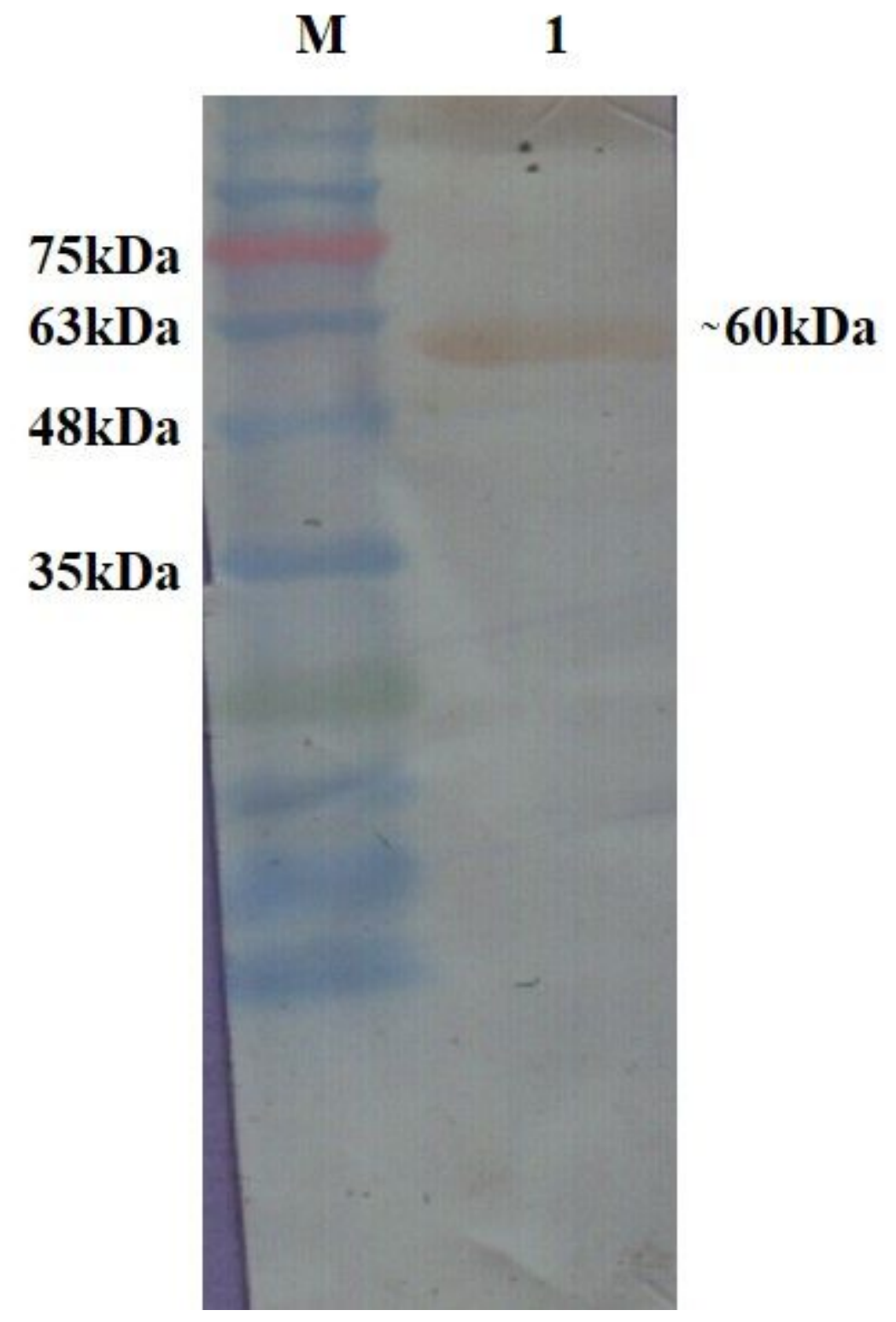

Figure 5

Western Blot analysis of rHSPD1 proteinby using IgG purified from hyperimmune sera raised against rHSPD1 in mice (Lane M: Pre-stained protein ladder; Lane 1: Immuno reactive rHSPD1) 


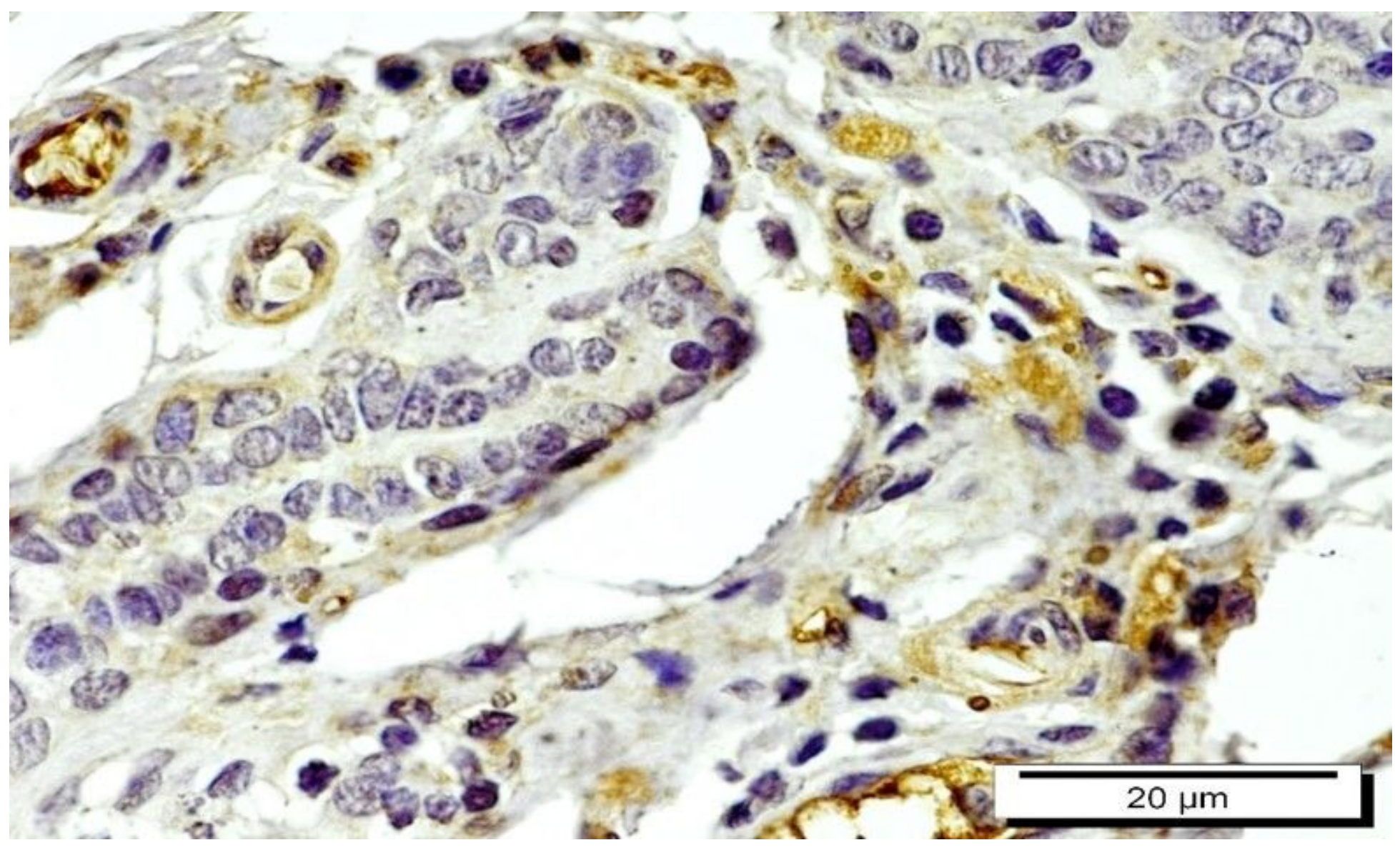

Figure 6

Immunolocalization of HSPD1 showing mild expression of HSPD1 within the cytoplasm in healthy tissue

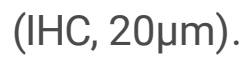




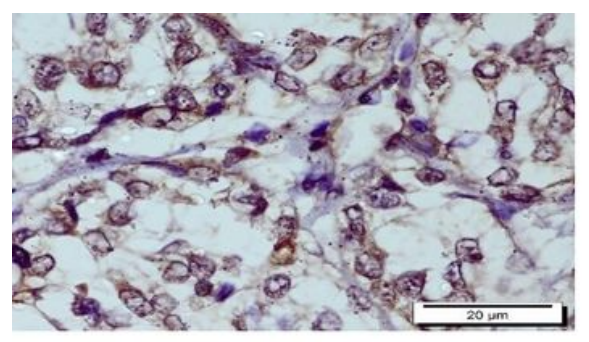

A

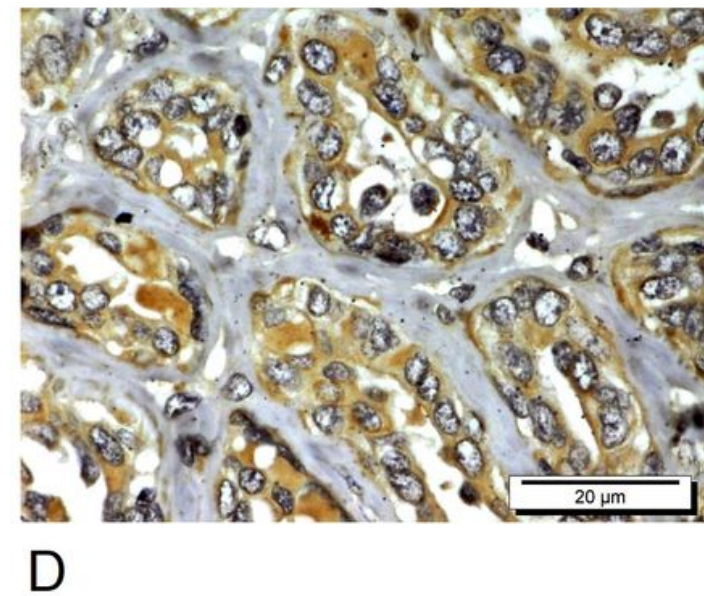

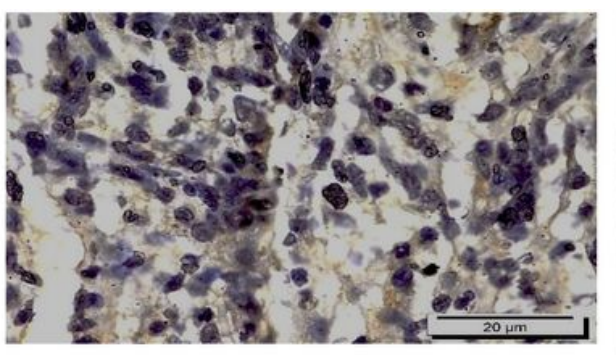

B

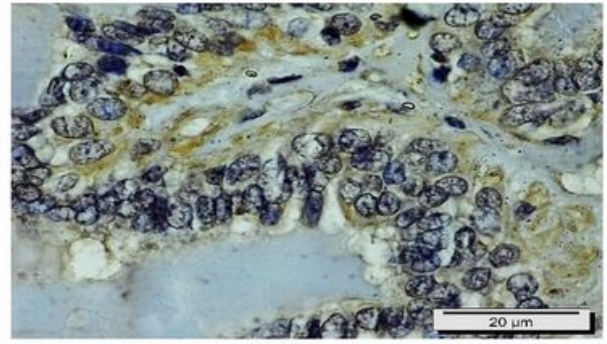

C

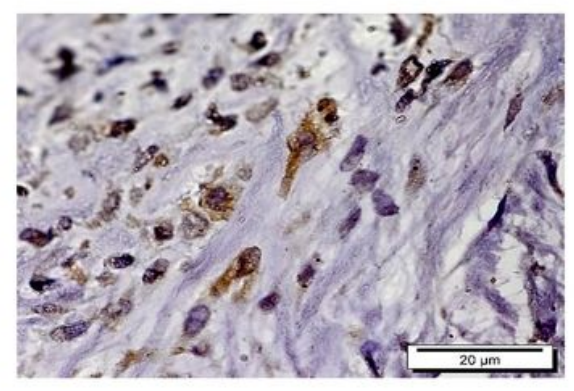

$\mathrm{E}$

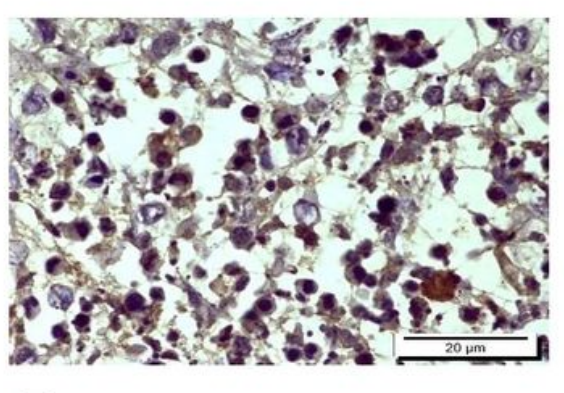

$\mathrm{F}$

\section{Figure 7}

(a) Anaplastic carcinoma: Immunolocalisation of HSPD1 showing intense expression of HSPD1 within the cytoplasm and mostly perinuclear area of the neoplastic epithelial cells (IHC, $20 \mu \mathrm{m})$; (b) Hemangiosarcoma: Immunolocalisation of HSPD1 showing mild expression of HSPD1 in the cytoplasm

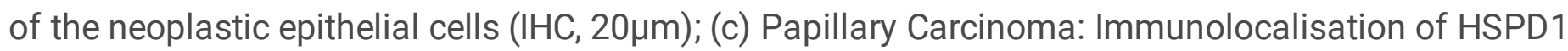

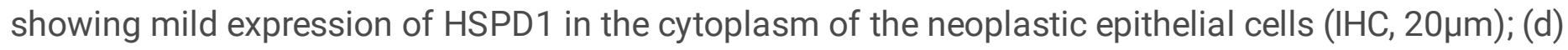
Complex Carcinoma: Immunolocalisation of HSPD1 showing intense expression of HSPD1 in the cytoplasm of the neoplastic epithelial cells which are arranged in nest (IHC, 20 $\mu \mathrm{m})$; (e) Mixed mammary tumor: Immunolocalisation of HSPD1 showing moderate expression of HSPD1 in the cytoplasm of the

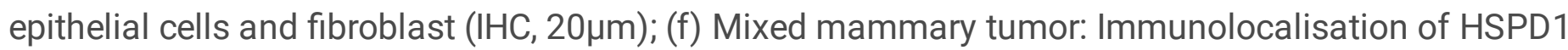
showing moderate expression of HSPD1 in the cytoplasm of the epithelial cells and fibroblast (IHC, $20 \mu \mathrm{m})$. 